\title{
A Comparative Assessment to Evaluate Enhanced External Counter Pulsation Effect on Physical Profile and Quality of Life in Diabetic and Nondiabetic Coronary Heart Disease Patients
}

\author{
Vikram Singh $^{1,2}$, Girija Kumari ${ }^{1,3}$, Bimal Chhajer ${ }^{2}$, Ashok Kumar Jhingan ${ }^{3}$, Saurabh Dahiya ${ }^{1 *}$ \\ ${ }^{1}$ Department of Pharmacy, Lingaya's University, Faridabad, India. \\ ${ }^{2}$ Department of Preventive Cardiology, SAAOL Heart Center, New Delhi, India. \\ ${ }^{3}$ Department of Medicine, Delhi Diabetes Research Center, New Delhi, India.
}

\begin{tabular}{|c|c|}
\hline ARTICLE INFO & ABSTRACT \\
\hline Artic & \multirow{11}{*}{$\begin{array}{l}\text { This study was conducted to assess the effect of Enhanced External Counter Pulsation (EECP) on physical profile and } \\
\text { Health-Related Quality of Life (HRQoL) in diabetic and nondiabetic Coronary Heart Disease (CHD) patients. This } \\
\text { pretest-posttest designed prospective study was conducted among } 163 \text { diabetic and nondiabetic coronary heart disease } \\
\text { patients in the SAAOL Heart Center, New Delhi. The physical profile of study subjects was assessed through Cooper's } \\
12 \text { minutes' walk test, Canadian Cardiovascular Society (CCS) angina scale and Medical Research Council (MRC) } \\
\text { dyspnea scale. The HRQoL of subjects was assessed using SF-36 (short form) and Seattle Angina Questionnaire } \\
\text { (SAQ) scale. A significant improvement was observed in blood pressure, heart rate, SpO }{ }_{2}, \mathrm{VO}_{2} \text { max, CCS angina } \\
\text { and MRC score in both the groups from baseline to } 12 \text { months. Significant improvement was also observed in both } \\
\text { the scales of HRQoL after EECP treatment at } 12 \text { months follow up in all the health domains of SF-36 \& SAQ scale } \\
\text { with special reference to angina severity and angina stability improvement. In conclusion, EECP is an effective } \\
\text { non-invasive therapy to treat diabetic and nondiabetic CHD patients. This non-invasive procedure may improve the } \\
\text { physical functional capacity, angina, dyspnea and overall HRQoL of diabetic and nondiabetic CHD patients. }\end{array}$} \\
\hline on: $24 / 02 / 2018$ & \\
\hline $17 / 05 / 2018$ & \\
\hline Available online: $29 / 06 / 2018$ & \\
\hline & \\
\hline Key words: & \\
\hline Coronary Heart Disease, & \\
\hline Diabetes Mellitus, Angina & \\
\hline Pectoris, Health-Related & \\
\hline $\begin{array}{l}\text { Quality of Life, Enhanced } \\
\text { External Counter Pulsation. }\end{array}$ & \\
\hline & \\
\hline
\end{tabular}

\section{INTRODUCTION}

Diabetes mellitus (DM) and coronary heart disease (CHD) has a close relationship with each other. Diabetes mellitus is an independent risk factor for CHD development (WHO, 2014). Patients with a history of diabetes are more likely to suffer from CHD compared to individuals without diabetes (Unnikrishnan et al., 2016). Diabetes mellitus and CHD are a major cause of mortality and morbidity globally (Fuster et al., 2010). CHD with DM patients has higher mortality rate due to uncontrolled diabetes which further causes several dysfunctions such as insulin resistance, atherosclerosis, and myocardial infarction. Indian and international registry data confirmed that $30-40$ percent DM patients have CHD in India (Ali et al., 2010). It is documented that

\section{${ }^{*}$ Corresponding Author}

Dr. Saurabh Dahiya, Ph.D, Professor and Head of Department,

Department of Pharmacy, Lingaya's University, India.

E-mail: saurabhdahiya@gmail.com more than 65 percent of cardiac deaths are due to diabetes mellitus in which 75-80 percent were especially due to CHD (Xavier et al., 2008; Moss et al., 1991).

Coronary heart disease can be treated by pharmacotherapy, Percutaneous Transluminal Coronary Angioplasty (PTCA), Coronary Artery Bypass Grafting (CABG), lifestyle management and non-invasive therapy known as Enhanced External Counter Pulsation (EECP) (Antman et al., 2008). Enhanced external counterpulsation is US-FDA approved non-invasive therapy for treatment of CHD patients, which are not suitable, unresponsive, and unwilling for PTCA and CABG procedures. Enhanced external counterpulsation therapy increases retrograde aortic blood flow during the diastolic phase of heart pumping. The mechanical device called EECP comprises sequential compression over legs, thighs, and buttocks to direct blood flow towards the heart when it is in the resting position. This treatment is recommended for 35 hours, one hour per day for 7 weeks ( 5 days in a week) (Arora $e t$ al., 1999; Kumar et al., 2017). 
Coronary heart disease and DM comorbidity deteriorate physical functional capacity and Health-Related Quality of Life (HR-QoL) of patients (Taghadosi et al., 2014). Bozorgi et al. and Singh et al. studies demonstrate that EECP may significantly improve health-related quality of life and exercise tolerance in diabetic and nondiabetic coronary heart disease patients (Bozorgi et al., 2014; Singh et al., 2018). The 12 minutes run or walk test was developed by Kenneth H. Cooper in 1968 to assess physical functioning capacity and to estimate $\mathrm{VO}_{2} \max$ (maximum oxygen uptake). $\mathrm{VO}_{2} \max$ is the maximum volume of oxygen consumption during an intense walk or exercise. The maximal aerobic or functional capacity is defined at the point at which oxygen consumption is high (Bandyopadhyay et al., 2015). Canadian Cardiovascular Society (CCS) angina scale is widely used to assess angina severity in coronary heart disease patients. This is a valid and reliable tool to evaluate angina severity, ranging from class I to class IV in CHD patients (Kaul et al., 2009). Medical Research Council (MRC) scale is widely used to assess breathlessness or dyspnea status in CHD patients. This scale comprises five segments that define the entire range of respiratory infirmity from none (Grade 1) to almost a complete incapacity (Grade 5) (Bestall et al., 1999).

The quality of life assessment of an individual is the status of well-being and feeling about their health and body functioning. SF-36 and SAQ are standard tools, which are used to assess general and disease specific health-related quality of life of CHD patients (Brazier et al., 1992). Thompson et al. study provides evidence for reliability and validity of SF-36 scale used to assess the quality of life in CHD patients (Thompson et al., 2003). Seattle angina questionnaire (SAQ) is a disease-specific quality of life assessment tool which has been shown to be a valid, reproducible and reliable tool to evaluate the treatment or intervention effectiveness in CHD patients (Dempster et al., 2000). The SAQ scale is widely used and its validity and reliability towards health status are demonstrated (Spertus et al., 1995). Enhanced external counterpulsation treatment helps in glycemic control which ultimately improve the health-related quality of life of diabetic patients and studies done by Linnemeier et al. and Ramasamy et al. validates the effectiveness of EECP in diabetes patients (Linnemeier et al., 2003; Ramasamy et al., 2015).

Our study was designed to assess the physical functioning profile and health-related quality of life of DM and CHD comorbidity patients and Non-DM CHD patients treated with EECP therapy.

\section{MATERIALS AND METHODS}

A pretest-posttest designed prospective study was done at Science and Art of Living (SAAOL) Heart Center, New Delhi among diabetic and nondiabetic CHD patients. A total of 212 subjects were enrolled in the study using consecutive sampling techniques from April 2016 to May 2017. The study subjects were divided into two groups. The first group was CHD patients with DM while the second group subjects were CHD patients without DM.

\section{Inclusion criteria}

Coronary heart disease patients with and without DM (patients having diabetes duration maximum 5 years) aged between
30 to 75 years, who did not willingly go for invasive treatments (Coronary Artery Bypass Grafting [CABG] and Percutaneous Trans Coronary Angioplasty [PTCA]) and agreed to participate in the study with valid written informed consent were enrolled.

\section{Exclusion criteria}

Patients having cardiac arrhythmia, coagulation disorder, deep vein thrombosis, vaso-occlusive disease, abnormal aortic aneurysm, cardiac valvular disorder, pregnancy, high blood pressure (higher than 180/110 $\mathrm{mmHg}$ ), foot wounds, dialysis history and unable to give valid written consent were excluded from the study.

\section{Study procedure}

The study was initiated with the screening of diabetic and nondiabetic CHD patients from the SAAOL Heart Center, New Delhi. A total of 300 subjects were screened. Out of 300 subjects, 88 were excluded from the study because they refused to participate, refused to give written consent and not willing to adhere 12 months study follow up. Finally, 212 study subjects were enrolled as they fulfilled all study eligibility criteria and agreed to 12 months of the study follow-up. After enrollment, the study subjects were allocated in CHD patient with DM (CHD + DM group) and CHD patients without DM (CHD group). After this, the demographic profile (age, gender, etc.), clinical history and physiological (systolic blood pressure, diastolic blood pressure, heart rate) profile, was done at baseline of all study subjects. Physical functioning capacity, $\mathrm{SpO}_{2}$ level, $\mathrm{VO}_{2}$ max, $\mathrm{CCS}$ angina status, and MRC dyspnea score assessed at baseline and followed at 6 and 12 months of study. Health-related quality of life of the study subjects was also assessed at baseline and followed at 6 and 12 months through SF-36 and Seattle Angina Questionnaire (SAQ).

\section{Measurements}

\section{Physiological profile assessment}

Systolic and diastolic blood pressure were measured at baseline, 6 and 12 months. Systolic blood pressure (SBP) and diastolic blood pressure (DBP) were recorded by qualified personnel through Diamond Clock Model blood pressure monitor. Heart rate \& $\mathrm{SpO}_{2}$ of all study subjects were assessed through pulse oximeter (ChoiceMMed MD300C2D) at baseline, 6 months, and 12 months.

\section{Physical functioning assessment}

Physical functional capacity was assessed through Cooper 12-minute walk test and $\mathrm{VO}_{2}$ max. In the Cooper 12-minute walk the study subjects were allowed to walk for 12 minutes on a flat track and a pedometer was used to measure the distance they covered within 12 minutes. $\mathrm{VO}_{2}$ max was used to assess the aerobic capacity of study subjects. It was calculated with the standard formula:

$\mathrm{VO}_{2} \max (\mathrm{ml} / \mathrm{kg} / \mathrm{min})=(22.351 \times$ distances covered in kilometers) -11.288 .

Angina status was assessed through the Canadian Cardiovascular Society (CCS) grading of angina pectoris. Breathlessness or dyspnea status was assessed by the Medical Research Council (MRC) scale. 


\section{Health-related quality of life (HRQoL) assessment}

The HRQoL of study subjects was assessed by using SF-36 and SAQ. HRQoL of CHD patients with and without DM was assessed through face to face interview and subjects were responding as they experienced problems related to mobility, normal working at work, study, personal care, leisure activities, family, pain, and depression/anxiety. Answers of the patients were recorded. Short Form-36 (SF-36) a multi-item scale comprising 36 questions was used to assess the general health-related quality of life of CHD patients with and without DM in eight health domains, namely; physical functioning, physical health problems, emotional problems, energy status, emotional well-being, body pain and general health status. The score generated for each health variable was from 0 to 100 , with 0 denoting the worst and 100 the best possible health outcomes. Seattle angina questionnaire was used to assess disease-specific quality of life and it measures 5 domains to assess EECP effectiveness in diabetic and nondiabetic CHD patients. SAQ domains were the physical constraint, angina stability, angina severity, treatment satisfaction, and perceived disease.

\section{EECP treatment}

The EECP, an electro-mechanical device, consists of three paired pneumatic cuffs applied to the lower leg, upper leg and buttocks. The cuffs of the device were inflated sequentially during diastole with maximum pressure $300 \mathrm{mmHg}$ and returning blood from the legs to the central circulation and producing aortic diastolic augmentation. This increases both venous return and cardiac output in coronary heart disease patients. In the next step, cuffs are deflated at end-diastole with reducing peripheral resistance and providing left ventricular supply.

The ECP PSK machine having the model; P-ECP/TI was used for the treatment of diabetic and nondiabetic coronary heart disease patients. Each subject underwent one hour per day EECP treatment for 35 days for 7 weeks ( 5 days in a week) with $280 \mathrm{mmHg}$ pressure. All study subjects were followed up for 12 months after completion of EECP therapy. Physical functional capacity through Cooper 12-minutes' walk test, $\mathrm{SpO}_{2}$ status, $\mathrm{VO}_{2}$ max, CCS angina severity and dyspnea score with health-related quality of life (SF-36, SAQ) were assessed at 6th and 12th months of study follow up (Bestall et al., 1999; Bandyopadhyay et al., 2015; Kaul et al., 2009).

\section{Ethical approval and consent}

Ethical permission for this study was obtained from the Institutional Ethics Committee of SAAOL Heart Center (Ref. No-IEC/SHRF/PhD/P-02/01.05.2016), New Delhi. Written informed consent was also taken from all study subjects before initiation of the study.

\section{Statistical analysis}

A senior Biostatistician analyzed the collected data using statistical package for the social sciences (SPSS) software version 21. Demographic and socioeconomic profile of the study subjects were compared using independent t-test. Descriptive analysis of mean, SD, independent t-test and paired sample t-test with $95 \%$ confidence interval and $p$-value $<0.05$ was considered statistically significant.

\section{RESULTS}

\section{Recruitment and response rates of the study subjects}

The study was initiated with the screening of total 300 diabetic and nondiabetic coronary heart disease patients from the study site. Out of 300 patients, 88 patients were excluded from the study because 26 patients refused to participate, 33 patients refused to give written consent and 19 patients did not agree to 12 months follow up. Finally, 212 study subjects were enrolled as they fulfilled all study eligibility criteria and agreed to 12 months of study follow up. After enrollment, the study subjects were allocated to coronary heart disease patients with diabetes mellitus and coronary heart disease patients without diabetes mellitus. Total 163 patients had completed the whole study with 12 months follow up, 49 patients did not complete whole study and follow up, so they were excluded from the final data analysis because of missing and incomplete data. Out of 49 patients, 24 patients were excluded from the CHD + DM group (13 patients left the treatment and migrated to abroad and 11 patients did not complete 12 months follow up) and 25 from the CHD group (9 patients migrated to different places, 16 patients did not complete 12 months follow up). Finally, the data of 163 patients were analyzed. Study outlines and response rates of the study subjects are summarized in the consort diagram (Table 1).

\section{Baseline demographic details with the medical history of diabetic and nondiabetic CHD groups}

A total of 163 diabetic and nondiabetic CHD patients completed the study (12 months follow up). Out of total 163 subjects; $\mathrm{n}=82$ subjects were in the diabetic CHD group (first group) and $n=81$ subjects were in the nondiabetic CHD group (second group). The total mean age of both the group was 59.6 \pm 9.5 (mean $\pm \mathrm{SD}$ ) years in which mean age of diabetic CHD subjects were $60.5 \pm 9.5$ (mean $\pm \mathrm{SD}$ ) and nondiabetic CHD subjects were $58.6 \pm 9.6$ (mean $\pm \mathrm{SD}$ ) years. The male subjects were $50.4 \%$ in diabetic CHD and $49.6 \%$ in nondiabetic CHD group. Females were much higher $(53.3 \%)$ in nondiabetic CHD as compared to diabetic CHD group (46.7\%). In this study, urban subjects were higher $(56.4 \%)$ in the diabetic CHD group and rural subjects were higher $(55.3 \%)$ in nondiabetic CHD group. A number of subjects have obesity $(63.1 \%)$ in nondiabetic CHD group as compared to diabetic CHD group (36.9\%). There were more hypertensive $(51.6 \%)$ patients in the diabetic CHD group as compared to nondiabetic CHD group (48.4\%).

The family history of heart disease $(62.5 \%)$ was higher in the diabetic CHD group as compared to nondiabetic CHD group (37.5\%), similarly, the family history diabetes $(83.3 \%)$ was also higher in the diabetic CHD group as compared to nondiabetic CHD subjects (16.7\%). The average diabetes duration in diabetic CHD group subjects was 4 years. Smoker patients were higher $(51.9 \%)$ in nondiabetic CHD group as compared to diabetic CHD group (48.1\%) and similarly, tobacco user was higher (83\%) in nondiabetic CHD group as compared to diabetic CHD group (17\%). Physical activity was higher $(53.2 \%)$ in nondiabetic CHD group as compared to diabetic CHD group (46.8\%). The non-vegetarian subjects were higher $(53.1 \%)$ in nondiabetic CHD group 
as compared to the diabetic CHD group (46.9\%). Medical history, such as myocardial infarction was higher in diabetes CHD group (54.9\%), similarly, the rate of PCI $(62.9 \%)$ and CABG (58.7\%) was also higher in diabetic CHD subjects. The history of single vessel CHD was similar in both the groups, but double vessel CHD patients were higher $(62.8 \%)$ in nondiabetic CHD subjects while triple vessel CHD was higher $(60 \%)$ in diabetic CHD subjects as compared to nondiabetic CHD subjects (40\%). The detail of the demographic profile and medical history of diabetic and nondiabetic CHD groups is described in Table 2.

Table 1: A systematic overview of the study.

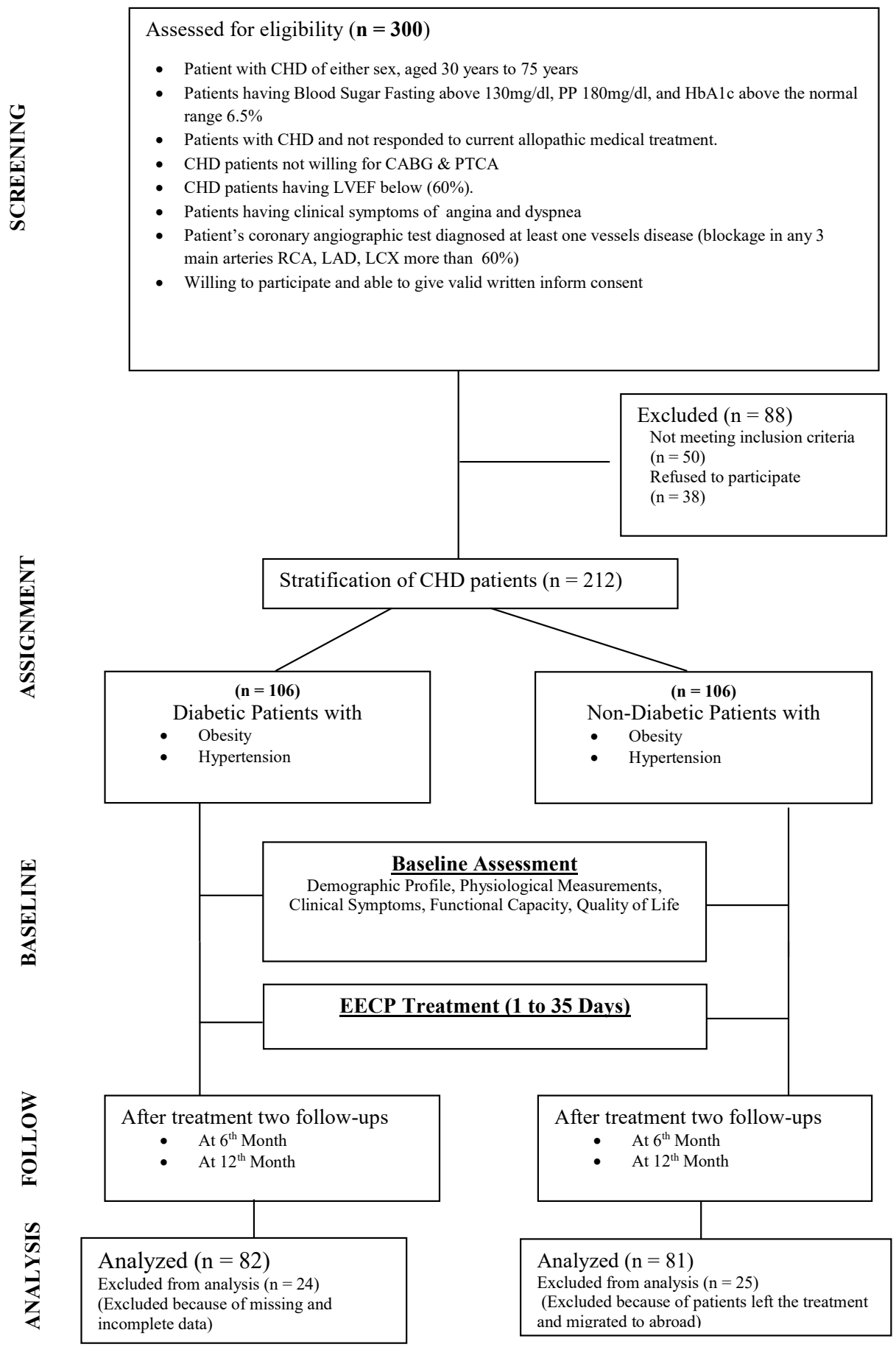


Table 2: Baseline demographic characteristics with medical history of diabetic \& nondiabetic CHD patients.

\begin{tabular}{|c|c|c|c|c|}
\hline Characteristics & Total $(n=163)$ & Diabetic CHD $(n=82)$ & Nondiabetic CHD $(n=81)$ & p-value \\
\hline Age (in years) & $59.6 \pm 9.5$ & $60.5 \pm 9.5$ & $58.6 \pm 9.6$ & 0.183 \\
\hline \multicolumn{5}{|c|}{ Gender } \\
\hline Male (n) & 133 & $67(50.4 \%)$ & $66(49.6 \%)$ & \multirow{2}{*}{0.714} \\
\hline Female (n) & 30 & $14(46.7 \%)$ & $16(53.3 \%)$ & \\
\hline \multicolumn{5}{|c|}{ Locality } \\
\hline Rural & 85 & $38(44.7 \%)$ & $47(55.3 \%)$ & \multirow{2}{*}{0.135} \\
\hline Urban & 78 & $44(56.4 \%)$ & $34(43.6 \%)$ & \\
\hline \multicolumn{5}{|c|}{ Obesity } \\
\hline No & 38 & $21(55.3 \%)$ & $17(44.7 \%)$ & \multirow{2}{*}{0.485} \\
\hline Yes & 125 & $46(36.9 \%)$ & $79(63.1 \%)$ & \\
\hline \multicolumn{5}{|c|}{ Hypertension } \\
\hline No & 72 & $35(48.6 \%)$ & $37(51.4 \%)$ & \multirow{2}{*}{0.753} \\
\hline Yes & 91 & $47(51.6 \%)$ & $44(48.4 \%)$ & \\
\hline \multicolumn{5}{|c|}{ Family History of Heart Disease } \\
\hline No & 115 & $52(45.2 \%)$ & $63(54.8 \%)$ & \multirow{2}{*}{0.733} \\
\hline Yes & 48 & $30(62.5 \%)$ & $18(37.5 \%)$ & \\
\hline \multicolumn{5}{|c|}{ Family History of Diabetes } \\
\hline No & 127 & $52(40.9 \%)$ & $75(59.1 \%)$ & \multirow{2}{*}{0.973} \\
\hline Yes & 36 & $30(83.3 \%)$ & $6(16.7 \%)$ & \\
\hline \multicolumn{5}{|c|}{ Smoking } \\
\hline No & 86 & $44(51.7 \%)$ & $42(48.3 \%)$ & \multirow{2}{*}{0.586} \\
\hline Yes & 77 & $37(48.1 \%)$ & $40(51.9 \%)$ & \\
\hline \multicolumn{5}{|c|}{ Tobacco } \\
\hline No & 138 & $78(56.5 \%)$ & $60(43.5 \%)$ & \multirow{2}{*}{$<0.0001$} \\
\hline Yes & 25 & $4(17.0 \%)$ & $21(83.0 \%)$ & \\
\hline \multicolumn{5}{|c|}{ Physical Activity } \\
\hline No & 101 & $53(52.5 \%)$ & $48(47.5 \%)$ & \multirow{2}{*}{0.480} \\
\hline Yes & 62 & $29(46.8 \%)$ & $33(53.2 \%)$ & \\
\hline \multicolumn{5}{|c|}{ Stress } \\
\hline Less & 61 & $31(50.8 \%)$ & $30(49.2 \%)$ & \multirow{2}{*}{0.919} \\
\hline More & 102 & $51(50.0 \%)$ & $51(50.0 \%)$ & \\
\hline \multicolumn{5}{|c|}{ Diet } \\
\hline Veg & 99 & $52(52.5 \%)$ & $47(47.5 \%)$ & \multirow{2}{*}{0.481} \\
\hline Non-Veg & 64 & $30(46.9 \%)$ & $34(53.1 \%)$ & \\
\hline \multicolumn{5}{|c|}{ Acute Myocardial Infarction (MI) } \\
\hline No & 92 & $43(46.7 \%)$ & $49(53.3 \%)$ & 0300 \\
\hline Yes & 71 & $39(54.9 \%)$ & $32(45.1 \%)$ & 0.500 \\
\hline & & Coronary Intervention (I & & \\
\hline No & 128 & $60(46.9 \%)$ & $68(53.1 \%)$ & 0094 \\
\hline Yes & 35 & $22(62.9 \%)$ & $13(37.1 \%)$ & 0.034 \\
\hline & & tery Bypass Graft (CAB & & \\
\hline No & 88 & $38(43.2 \%)$ & $50(56.8 \%)$ & مه \\
\hline Yes & 75 & $44(58.7 \%)$ & $31(41.3 \%)$ & \\
\hline & & e (Vessel) of CHD & & \\
\hline Single & 70 & $35(50 \%)$ & $35(50 \%)$ & \\
\hline Double & 43 & $16(37.2 \%)$ & $27(62.8 \%)$ & 0.058 \\
\hline Triple & 50 & $30(60 \%)$ & $20(40 \%)$ & \\
\hline
\end{tabular}


Table 3: Baseline \& follow up results of a physiological profile in diabetic \& nondiabetic CHD patients.

\begin{tabular}{|c|c|c|c|c|c|c|}
\hline \multirow[t]{2}{*}{ Variables } & & \multicolumn{2}{|c|}{ Diabetic CHD } & \multicolumn{2}{|c|}{ Nondiabetic CHD } & \multirow[t]{2}{*}{ p-value } \\
\hline & & Mean & SD & Mean & SD & \\
\hline \multicolumn{7}{|c|}{ SBP } \\
\hline Baseline & & 131.2 & 15.1 & 132.6 & 16.1 & 0.575 \\
\hline 6 Month & & 124.3 & 10.4 & 125.6 & 12.2 & 0.43 \\
\hline 12 Month & & 128 & 12.2 & 129.3 & 14.0 & 0.558 \\
\hline \multirow{2}{*}{ p-value } & Baseline - 6 Month & \multicolumn{2}{|c|}{$<0.001$} & \multicolumn{2}{|c|}{$<0.001$} & \\
\hline & Baseline - 12 Month & \multicolumn{2}{|c|}{$<0.001$} & \multicolumn{2}{|c|}{$<0.001$} & \\
\hline \multicolumn{7}{|c|}{ DBP } \\
\hline Baseline & & 84.1 & 7.7 & 83.8 & 7.8 & 0.793 \\
\hline 6 Month & & 79.1 & 6.1 & 78.6 & 6.8 & 0.621 \\
\hline 12 Month & & 81.9 & 6.5 & 83.5 & 6.9 & 0.126 \\
\hline \multirow{2}{*}{ p-value } & Baseline - 6 Month & \multicolumn{2}{|c|}{$<0.001$} & \multicolumn{2}{|c|}{$<0.001$} & \\
\hline & Baseline - 12 Month & \multicolumn{2}{|c|}{0.041} & \multicolumn{2}{|c|}{0.658} & \\
\hline \multicolumn{7}{|c|}{ Heart Rate } \\
\hline Baseline & & 79.3 & 6.1 & 78.4 & 6.3 & 0.263 \\
\hline 6 Month & & 76.7 & 5.0 & 75.5 & 5.1 & 0.257 \\
\hline 12 Month & & 76.9 & 3.9 & 78.1 & 5.3 & 0.057 \\
\hline \multirow{2}{*}{$\mathrm{p}$-value } & Baseline - 6 Month & \multicolumn{2}{|c|}{$<0.001$} & \multicolumn{2}{|c|}{$<0.001$} & \\
\hline & Baseline - 12 Month & \multicolumn{2}{|c|}{$<0.001$} & \multicolumn{2}{|c|}{0.934} & \\
\hline \multicolumn{7}{|c|}{$\mathrm{SpO}_{2}$ status } \\
\hline Baseline & & 96.0 & 1.5 & 96.5 & 1.6 & 0.343 \\
\hline 6 Month & & 98.2 & 1.7 & 98.4 & 1.8 & 0.938 \\
\hline 12 Month & & 99.1 & 1.0 & 98.9 & 1.1 & 0.157 \\
\hline \multirow{2}{*}{ p-value } & Baseline - 6 Month & \multicolumn{2}{|c|}{$<0.0001$} & \multicolumn{2}{|c|}{$<0.0001$} & \\
\hline & Baseline - 12 Month & \multicolumn{2}{|c|}{$<0.0001$} & \multicolumn{2}{|c|}{$<0.0001$} & \\
\hline
\end{tabular}

\section{Effect of EECP on physiological parameters and physical capacity in diabetic and nondiabetic CHD patients}

The physiological parameters consist systolic blood pressure, diastolic blood pressure, heart rate and $\mathrm{SpO}_{2}$ did not show any significant change between the groups from baseline to 12 months using independent t-test. A significant change has been observed in systolic blood pressure, diastolic blood pressure, heart rate and $\mathrm{SpO}_{2}$ level from baseline to 6 months in diabetic CHD patients and similar outcomes sustained till 12 months in systolic blood pressure and $\mathrm{SpO}_{2}$ level in both the groups. The detailed information has been given in Table 3 .

A significant change was observed in dyspnea score at baseline in male using MRC scale but at 6 months and 12 months follow up no statistical change was observed. There was no significant change between the group in Cooper 12 minute walk test (walking distance), $\mathrm{VO}_{2} \max$ and $\mathrm{CCS}$ angina classifications were observed in diabetic and nondiabetic CHD group patients. The details of findings have been given in Table 4 .

\section{Effect of EECP on health-related quality of life (HRQoL) in diabetic and nondiabetic CHD patients}

A significant improvement was observed in all SF-36 scale domains from baseline to 12 months with significant pvalues $<0.001$. The significant changes were also observed in, energy level, social functioning, and general health after 6 months of EECP treatment between the groups but these differences did not maintain until 12 months. The difference between the groups was assessed using independent t-test. The details of SF-36 study outcomes have been given in Table 5 .

All domains of SAQ scale yielded significant improvement within both (diabetic \& nondiabetic CHD) groups from baseline to 12 months as assessed through paired sample t-test. Significant improvement in treatment satisfaction and disease perception after the 12 months of EECP treatment has been observed in both diabetic and nondiabetic CHD groups. There was no significant difference observed between both the groups from baseline to 6th months and 12th months of EECP treatment, assessed through the independent t-test. The details of SAQ results have been given in Table 6.

\section{DISCUSSION}

The aim of the present study was to assess the effect of EECP therapy on the physical functional status and health-related quality of life in diabetic and nondiabetic CHD patients. Our study demonstrates significant improvement in blood pressure, heart rate, and $\mathrm{SpO}_{2}$ level after EECP treatment in both groups. Beck et $a l$. and Casey et al. study also indicated that EECP may be useful as an adjuvant therapy for improving functional capacity in coronary heart disease patients through reductions in blood pressure and improvement in myocardial oxygen demand for better physical functioning (Beck et al., 2015; Casey et al., 2011). Diabetic and nondiabetic CHD patients demonstrate significant improvement 
in walking status, exercise tolerance with increment in $\mathrm{VO}_{2}$ max levels after EECP therapy. Similarly, studies done by Feldman et al. and Urano et al. have supported the findings of the present study (Feldman et al., 2006; Urano et al., 2001).

Table 4: EECP Treatment effect on Cooper's 12-Minutes' walk test with $\mathrm{VO}_{2}$ max, Angina \& Dyspnea status at Baseline, 6th month and 12th month in CHD \& DM with CHD patients.

\begin{tabular}{|c|c|c|c|c|}
\hline Time & Variables & CHD (n = 81) & $\mathrm{DM}+\mathrm{CHD}(\mathrm{n}=82)$ & p-value \\
\hline & Male & $\mathrm{n}=67$ & $\mathrm{n}=66$ & \\
\hline \multirow[t]{4}{*}{ Baseline } & 12 Minute test (Distance) & $1449.7 \pm 124.51$ & $1491.2 \pm 173.63$ & 0.115 \\
\hline & $\mathrm{VO}_{2} \max$ & $21.12 \pm 2.72$ & $22.05 \pm 3.83$ & 0.117 \\
\hline & CCS Class & $2.8 \pm 0.24$ & $2.9 \pm 0.16$ & 0.678 \\
\hline & Dyspnea & $2.8 \pm 0.15$ & $3.2 \pm 0.21$ & 0.042 \\
\hline \multirow{4}{*}{6 Month } & 12 Minute test (Distance) & $2103.2 \pm 222.1$ & $2116.9 \pm 224.8$ & 0.725 \\
\hline & $\mathrm{VO}_{2} \max$ & $35.73 \pm 4.9$ & $36.04 \pm 5.02652$ & 0.727 \\
\hline & CCS Class & $2.1 \pm 0.12$ & $2.2 \pm 0.23$ & 0.326 \\
\hline & Dyspnea & $2.6 \pm 0.14$ & $2.7 \pm 0.28$ & 0.468 \\
\hline \multirow[t]{5}{*}{12 Month } & 12 Minute test (Distance) & $2100.4 \pm 384.3$ & $2050.6 \pm 413.0$ & 0.472 \\
\hline & $\mathrm{VO}_{2} \max$ & $35.67 \pm 8.5$ & $34.56 \pm 9.2$ & 0.474 \\
\hline & CCS Class & $2.3 \pm 0.25$ & $2.2 \pm 0.23$ & 0.185 \\
\hline & Dyspnea & $2.2 \pm 0.23$ & $2.1 \pm 0.12$ & 0.325 \\
\hline & Female & $\mathrm{n}=14$ & $\mathrm{n}=16$ & \\
\hline \multirow[t]{4}{*}{ Baseline } & 12 Minute test (Distance) & $1261.4 \pm 107.12$ & $1237.5 \pm 57.44$ & 0.444 \\
\hline & $\mathrm{VO}_{2} \max$ & $16.91 \pm 2.39$ & $16.38 \pm 1.28$ & 0.464 \\
\hline & CCS Class & $3.0 \pm 0.01$ & $2.9 \pm 0.14$ & 0.423 \\
\hline & Dyspnea & $2.8 \pm 0.14$ & $2.9 \pm 0.07$ & 0.712 \\
\hline \multirow[t]{4}{*}{6 Month } & 12 Minute test (Distance) & $1682.8 \pm 261.45$ & $1654.3 \pm 208.29$ & 0.742 \\
\hline & $\mathrm{VO}_{2} \max$ & $26.33 \pm 5.84$ & $25.7 \pm 4.65$ & 0.746 \\
\hline & CCS Class & $2.4 \pm 0.28$ & $2.1 \pm 0.14$ & 0.312 \\
\hline & Dyspnea & $2.4 \pm 0.21$ & $2.3 \pm 0.42$ & 0.698 \\
\hline \multirow[t]{4}{*}{12 Month } & 12 Minute test (Distance) & $1679.2 \pm 389.01$ & $1893.7 \pm 367.60$ & 0.132 \\
\hline & $\mathrm{VO}_{2} \max$ & $26.25 \pm 8.69$ & $31.05 \pm 8.21$ & 0.134 \\
\hline & CCS Class & $2.1 \pm 0.29$ & $2.0 \pm 0.10$ & 0.667 \\
\hline & Dyspnea & $1.9 \pm 0.05$ & $1.5 \pm 0.70$ & 0.059 \\
\hline
\end{tabular}

In the present study, a significant improvement was observed in the physical functional capacity in coronary heart disease patients after EECP therapy and similar findings have been reported by Rampengan et al. and May et al. in their study (Rampengan et al., 2015; May et al., 2015). Before EECP treatment our study subjects were mostly poor in physical functional capacity but after EECP treatment they significantly improved. A significant effect of EECP treatment on CCS angina class and dyspnea improvement has been observed in this study in diabetic and nondiabetic coronary heart disease patients. Studies done by Chung-Kuan et al. \& Ziaeirad et al. (Chung-Kuan et al., 2014; Ziaeirad et al., 2012) have shown similar findings. The results of our study are in agreement with Petterson et al. and Loh et al. studies which have shown significant results on the effectiveness of EECP in patients with angina (Pettersson et al., 2006; Loh et al., 2006). Soran et al. study also reported similar findings in the present study (Soran et al., 2006).

A significant improvement has been observed in healthrelated quality of life using SF-36 and SAQ scale and Arora et al. have reported similar findings (Arora, 1999). A significant improvement has been observed in all SAQ domains using the paired t-test to assess differences within groups from baseline to
12 months showed significant changes in both groups after EECP treatment. These results demonstrate similar outcomes in diabetic and nondiabetic CHD patients treated with EECP therapy. Our study shows improvement in the quality of life in diabetic and nondiabetic CHD patients and supported by the research done by Linnermeir et al. and Michaels et al. (Linnemeier et al., 2003; Michaels et al., 2004).

A significant improvement was observed in the quality of life with similar clinical outcomes of Ziaeirad et al. in diabetic and nondiabetic coronary heart disease patients. The author reported significant improvements in quality of life of coronary heart patients in all domains of SF-36, a standard questionnaire of quality of life assessment (Ziaeirad et al., 2012). SF-36 tool used by Arora et al. showed significant results on physical activity, general health with reduction of bodily pain and similarly Manchanda et al. indicated good results of EECP in respect of the improvement in the quality of life for heart patients (Manchanda et al., 2007). In the present study, overall, general health status had improved, and it is supported with the data of earlier studies to demonstrate the clinical significance of EECP health-related quality of life of coronary heart disease patients (Wu et al., 2012). 
Table 5: SF-36 Baseline \& follow up results of diabetic \& nondiabetic CHD patients.

\begin{tabular}{|c|c|c|c|c|}
\hline & & Nondiabetic CHD & Diabetic CHD & p-value \\
\hline \multicolumn{5}{|c|}{ Physical Functioning } \\
\hline Baseline & & $59.5 \pm 9.6$ & $60.1 \pm 7.8$ & 0.694 \\
\hline 6 Month & & $63.3 \pm 8.6$ & $60.6 \pm 10.3$ & 0.082 \\
\hline 12 Month & & $82.9 \pm 6.0$ & $82.7 \pm 6.4$ & 0.774 \\
\hline \multirow{2}{*}{$\mathrm{p}$-value } & Baseline - 6 Month & 0.020 & 0.702 & \\
\hline & Baseline - 12 Month & $<0.0001$ & $<0.0001$ & \\
\hline \multicolumn{5}{|c|}{ Role Limitation due to physical health } \\
\hline Baseline & & $34.8 \pm 23.6$ & $38.1 \pm 26.4$ & 0.409 \\
\hline 6 Month & & $50.6 \pm 27.1$ & $43.9 \pm 21.7$ & 0.083 \\
\hline 12 Month & & $62.9 \pm 24.9$ & $60.7 \pm 24.1$ & 0.543 \\
\hline \multirow{2}{*}{ p-value } & Baseline - 6 Month & $<0.0001$ & 0.147 & \\
\hline & Baseline - 12 Month & $<0.0001$ & $<0.0001$ & \\
\hline \multicolumn{5}{|c|}{ Role Limitation due to emotional problems } \\
\hline Baseline & & $37.0 \pm 29.3$ & $37.4 \pm 29.1$ & 0.938 \\
\hline 6 Month & & $44.4 \pm 29.8$ & $52.0 \pm 27.7$ & 0.095 \\
\hline 12 Month & & $59.3 \pm 27.9$ & $60.2 \pm 31.2$ & 0.848 \\
\hline \multirow{2}{*}{ p-value } & Baseline - 6 Month & 0.121 & 0.001 & \\
\hline & Baseline - 12 Month & $<0.0001$ & $<0.0001$ & \\
\hline \multicolumn{5}{|c|}{ Energy/Fatigue } \\
\hline Baseline & & $51.2 \pm 12.1$ & $45.5 \pm 9.1$ & 0.001 \\
\hline 6 Month & & $57.0 \pm 12.9$ & $52.1 \pm 12.3$ & 0.012 \\
\hline 12 Month & & $65.7 \pm 16.1$ & $67.9 \pm 16.8$ & 0.393 \\
\hline \multirow{2}{*}{ p-value } & Baseline - 6 Month & 0.004 & $<0.0001$ & \\
\hline & Baseline - 12 Month & $<0.0001$ & $<0.0001$ & \\
\hline \multicolumn{5}{|c|}{ Emotional Wellbeing } \\
\hline Baseline & & $64.6 \pm 12.1$ & $63.8 \pm 11.7$ & 0.676 \\
\hline 6 Month & & $72.2 \pm 20.3$ & $68.1 \pm 10.3$ & 0.106 \\
\hline 12 Month & & $73.3 \pm 22.4$ & $72.9 \pm 21.6$ & 0.875 \\
\hline \multirow{2}{*}{ p-value } & Baseline - 6 Month & 0.002 & 0.010 & \\
\hline & Baseline - 12 Month & $<0.0001$ & 0.001 & \\
\hline \multicolumn{5}{|c|}{ Social Functioning } \\
\hline Baseline & & $41.7 \pm 19.6$ & $42.4 \pm 13.9$ & 0.790 \\
\hline 6 Month & & $50.3 \pm 15.7$ & $44.4 \pm 20.9$ & 0.042 \\
\hline 12 Month & & $84.4 \pm 8.9$ & $84.6 \pm 9.1$ & 0.979 \\
\hline \multirow{2}{*}{ p-value } & Baseline - 6 Month & 0.005 & 0.485 & \\
\hline & Baseline - 12 Month & $<0.0001$ & $<0.0001$ & \\
\hline & & Bodily pain & & \\
\hline Baseline & & $66.5 \pm 15.9$ & $66.2 \pm 16.7$ & 0.909 \\
\hline 6 Month & & $74.2 \pm 13.4$ & $76.4 \pm 16.1$ & 0.343 \\
\hline 12 Month & & $78.5 \pm 19.3$ & $77.0 \pm 12.7$ & 0.589 \\
\hline \multirow{2}{*}{ p-value } & Baseline - 6 Month & $<0.0001$ & $<0.0001$ & \\
\hline & Baseline - 12 Month & $<0.0001$ & $<0.0001$ & \\
\hline \multicolumn{5}{|c|}{ General Health } \\
\hline Baseline & & $55.1 \pm 10.4$ & $55.3 \pm 12.1$ & 0.919 \\
\hline 6 Month & & $63.8 \pm 9.9$ & $57.3 \pm 9.2$ & $<0.0001$ \\
\hline 12 Month & & $88.1 \pm 7.3$ & $88.3 \pm 7.1$ & 0.899 \\
\hline \multirow{2}{*}{ p-value } & Baseline - 6 Month & $<0.0001$ & 0.257 & \\
\hline & Baseline - 12 Month & $<0.0001$ & $<0.0001$ & \\
\hline
\end{tabular}


Table 6: SAQ - Baseline \& follow up results of diabetic \& nondiabetic CHD patients.

\begin{tabular}{|c|c|c|c|c|}
\hline & & Nondiabetic CHD & Diabetic CHD & p-value \\
\hline \multicolumn{5}{|c|}{ Physical Limitation } \\
\hline Baseline & & $23.9 \pm 11.1$ & $23.7 \pm 12.7$ & 0.911 \\
\hline 6 Month & & $39.1 \pm 10.7$ & $38.4 \pm 12.6$ & 0.729 \\
\hline 12 Month & & $56.2 \pm 10.1$ & $54.3 \pm 10.9$ & 0.269 \\
\hline \multirow{2}{*}{ p-value } & Baseline - 6 Month & $<0.0001$ & $<0.0001$ & \\
\hline & Baseline - 12 Month & $<0.0001$ & $<0.0001$ & \\
\hline \multicolumn{5}{|c|}{ Angina Stability } \\
\hline Baseline & & $26.4 \pm 18.4$ & $27.8 \pm 20.1$ & 0.647 \\
\hline 6 Month & & $51.1 \pm 18.1$ & $52.2 \pm 18.9$ & 0.704 \\
\hline 12 Month & & $74.8 \pm 16.9$ & $74.1 \pm 17.2$ & 0.807 \\
\hline \multirow{2}{*}{ p-value } & Baseline - 6 Month & $<0.0001$ & $<0.0001$ & \\
\hline & Baseline - 12 Month & $<0.0001$ & $<0.0001$ & \\
\hline \multicolumn{5}{|c|}{ Angina Severity } \\
\hline Baseline & & $25.4 \pm 13.4$ & $24.4 \pm 12.2$ & 0.604 \\
\hline 6 Month & & $36.5 \pm 14.4$ & $33.6 \pm 12.1$ & 0.169 \\
\hline 12 Month & & $54.8 \pm 13.3$ & $54.6 \pm 13.7$ & 0.932 \\
\hline \multirow{2}{*}{ p-value } & Baseline - 6 Month & $<0.0001$ & $<0.0001$ & \\
\hline & Baseline - 12 Month & $<0.0001$ & $<0.0001$ & \\
\hline \multicolumn{5}{|c|}{ Treatment Satisfaction } \\
\hline Baseline & & $49.7 \pm 30.7$ & $51.5 \pm 23.0$ & 0.667 \\
\hline 6 Month & & $55.2 \pm 26.5$ & $52.1 \pm 29.4$ & 0.395 \\
\hline 12 Month & & $76.8 \pm 23.6$ & $76.2 \pm 22.5$ & 0.861 \\
\hline \multirow{2}{*}{ p-value } & Baseline - 6 Month & 0.210 & 0.846 & \\
\hline & Baseline - 12 Month & $<0.0001$ & $<0.0001$ & \\
\hline \multicolumn{5}{|c|}{ Disease Perception } \\
\hline Baseline & & $51.8 \pm 34.1$ & $50.6 \pm 33.7$ & 0.816 \\
\hline 6 Month & & $58.3 \pm 23.9$ & $54.2 \pm 24.8$ & 0.293 \\
\hline 12 Month & & $76.5 \pm 25.2$ & $74.3 \pm 26.5$ & 0.590 \\
\hline \multirow{2}{*}{ p-value } & Baseline - 6 Month & 0.170 & 0.409 & \\
\hline & Baseline - 12 Month & $<0.0001$ & $<0.0001$ & \\
\hline
\end{tabular}

Our study showed similar results in diabetic and nondiabetic CHD patients and no major significant difference has been observed in diabetic and nondiabetic CHD group using independent t-test in physical functional capacity and healthrelated quality of life. Eslamian et al. concluded that EECP therapy can improve the angina pectoris stability and severity of disease perception (Eslamian et al., 2013). Similarly, our study demonstrates that both diabetic and nondiabetic coronary heart disease patients get good results in almost all domains of SAQ quality of life assessment scale.

Enhanced external counterpulsation is an effective treatment method for diabetes management and diabetes complication including CHD. It helps in lowering blood glucose level of alerting transport of insulin into skeletal muscle and thereby help in the glycemic control and ultimately improve the healthrelated quality of life of diabetic CHD patients. Linnemeier et al., Martin et al., and Ramasamy et al. studies validate the effectiveness of EECP towards glycemic control in DM patients (Linnemeier et al., 2003; Martin et al., 2012; Ramasamy et al., 2015).

Our study reveals that EECP significantly improves the health-related quality of life and remained high for the following one year in diabetic and nondiabetic CHD patients. Jorgensen et al. study demonstrated that the effect of EECP on quality of life sustained for three years in CHD patients (Jorgensen et al., 2013). Hence, there is further need to conduct a multicentric randomized controlled trial to assess long-term effects of enhanced external counterpulsation on health-related quality of life in diabetic CHD patients. 


\section{CONCLUSIONS}

The results of the present study conclude that enhanced external counterpulsation therapy may improve walking capacity, maximal oxygen uptake and peripheral capillary oxygen saturation of diabetic and nondiabetic CHD patients. This non-invasive procedure also significantly improves the clinical symptoms (angina \& dyspnea) of CHD patients with and without DM. Enhanced external counterpulsation therapy may also improve overall health-related quality of life of diabetic and nondiabetic CHD patients, including angina severity, angina stability, and general health. In the summary, enhanced external counterpulsation therapy is safe, well tolerated and proved to be an effective, non-invasive therapy to treat diabetic and nondiabetic CHD patients. It may improve the physical profile and quality of life in both diabetic and nondiabetic CHD patients equally. There is further need of multicentric randomized controlled trials to assess long-term effects of enhanced external counterpulsation on health-related quality of life in a larger population of CHD with DM patients.

\section{CONFLICT OF INTEREST}

The authors of this study have no conflict of interest associated with material presented in this paper.

\section{ACKNOWLEDGMENT}

We are very thankful to the research team of the SAAOL Heart Center, New Delhi to complete this research study.

\section{REFERENCES}

Ali M K, Narayan K M, Tandon N. Diabetes and coronary heart disease: current perspectives. Indian J. Med, 2010; 132(5):584-597.

Antman EM, Selwyn AP, Braunwald E, Harrison LJ. 2008. Principles of internal medicine, cardiovascular diseases. Trans. In: Saadat N, Rasooli MR. 1st ed. Tehran: Andishe rafie publication company.

Arora RR, Chou TM, Jain D, Fleishman B, Crawford L, McKiernan T, et al. The multicenter study of enhanced external counter pulsation (MUST-EECP): Effect of EECP on exercise-induced myocardial ischemia and anginal episodes. J Am Coll Cardiol, 1999; 33(7):1833-1840.

Bandyopadhyay A. Validity of cooper's 12-minute run test for estimation of maximum oxygen uptake in male university students. Biol Sport, 2015; 32(1):59-63.

Beck DT, Casey DP, Martin JS, et al. Enhanced External Counter pulsation Reduces Indices of Central Blood Pressure and Myocardial Oxygen Demand in Patients with Left Ventricular Dysfunction. Clin Exp Pharmacol Physiol, 2015; 42(4):315-320.

Bestall C, Paul EA, Garrod R, Garnham R, et al. Usefulness of the Medical Research Council (MRC) dyspnoea scale as a measure of disability in patients with chronic obstructive pulmonary disease. Thorax, 1999; 54(7):581-586.

Bozorgi A, Mehrabi Nasab E, Sardari A, Nejatian M, Nasirpour S, Sadeghi S. Effect of Enhanced External Counterpulsation (EECP) on Exercise Time Duration and Functional Capacity in Patients with Refractory Angina Pectoris. J The Univ Heart Ctr, 2014; 9(1):33-37.

Brazier JE, Harper NM, Jones A, et al. Validating the SF-36 health questionnaire: A new outcome measure for primary care. BMJ, 1992; 305(6846):161-164.

Casey DP, Beck DT, Nichols WW, Conti CR, Choi CY, Khuddus MA, Braith RW. Effects of EECP on arterial stiffness and myocardial oxygen demand in patients with chronic angina pectoris. Am J Cardiol, 2011; 107(10):1466-1472.

Chung-Kuan Wu, Huei-Fong Hung, et al. The immediate and one-year outcomes of dialysis patients with refractory angina treated by enhanced external counter pulsation. Clin. Nephrol, 2014; 82(1):34-40.

Dempster M, Donnelly M. Measuring the health related quality of life of people with ischemic heart disease. Heart, 2000; 83(6):641-644.

Eslamian F, Aslanabadi N, Mahmoudian B, Shakouri SK Therapeutic effects of Enhanced External Counter Pulsation (EECP) on clinical symptoms, echocardiographic measurements, perfusion scan parameters and exercise tolerance test in coronary artery disease patients with refractory angina. Int J Med Sci Public Health, 2013; 2(2):187-195.

Feldman AM, Silver MA, Francis GS, et al. Enhanced External Counter pulsation Improves Exercise Tolerance in Patients with Chronic Heart Failure. J Am Coll Cardiol, 2006; 48(6):1199-1206.

Fuster V, Kelly BB. Board for global health. Promoting cardiovascular health in developing world: A critical challenge to achieve global health. Washington, DC: Inst of Med; 2010.

Global status report on non-communicable diseases, Geneva; Switzerland. WHO, 2014.

Jorgensen MT, May O. Improvement of angina, quality of life, and working capacity after enhanced external counterpulsation. Ugeskr Laeger, 2013; 175(3):114-116.

Kaul P, Naylor CD, Armstrong PW, et al. Assessment of activity status and survival according to the canadian cardiovascular society angina classification. Can J Cardiol, 2009; 25(7):225-231.

Kumar S, Lahiri TK. Enhanced external counterpulsation as an effective nonsurgical solution for ischemic heart disease patients. Heart India, 2017; 5(2):55-60.

Linnemeier G, Rutter MK, Barsness G, Kennard ED, Nesto RW. Enhanced external counter pulsation for the relief of angina in patients with diabetes: safety, efficacy and 1-year clinical outcomes. Am Heart J, 2003; 146(3):453-458.

Linnemeier G, Rutter MK, Barsness G. Enhanced External Counterpulsation for the relief of angina in patients with diabetes: Safety, efficacy and 1-year clinical outcomes. Am Heart J, 2003; 146(3):453-458.

Loh PH, Louis AA, Windram J. The immediate and long-term outcome of enhanced external counterpulsation in treatment of chronic stable refractory angina. J. Intern. Med., 2006; 259(3):276-284.

Manchanda A, Soran O. Enhanced external counter pulsation and future directions: step beyond medical management for patients with angina and heart failure. J Am Coll Cardiol, 2007; 50(16):1523-1531.

Martin JS, Beck DT, Aranda JM, Braith RW. Enhanced external counterpulsation improves peripheral artery function and glucose tolerance in subjects with abnormal glucose tolerance. J Appl Physiol, 2012; 112: 868-876.

May O, Lynggaard V, Mortensen JC, Malczynski J. Scand Enhanced External Counter pulsation - Effect on Angina Pectoris, QoL and Exercise Capacity after 1 Year. Cardiovasc J, 2015; 49(1):1-6.

Michaels AD, Linnemeier G, Soran O, et al. Two-year outcomes after enhanced external counter pulsation for stable angina pectoris (from the international EECP patient registry IEPR). Am J Cardiol, 2004; 93:461464.

Moss SE, Klein R, Klein BE. Cause-specific mortality in a population-based study of diabetes. Am J Public Health, 1991; 81(9):11581162.

Pettersson T, Bondesson S, Cojocaru D, Ohlsson O, Wackenfors A, Edvinsson L. One year follow-up of patients with refractory angina pectoris treated with enhanced external counterpulsation. BMC Cardiovasc Disord., 2006; 6(28):1-7.

Ramasamy S, Sivakadaksham N, Pradeep G, et al. Is the benefits of enhanced external counterpulsation in patient with moderate left ventricular dysfunction independent of diabetes? J Am Coll Cardiol, 2015; 66(16 supp):C142.

Rampengan SH, Prihartono J, Siagian M, et al. The Effect of Enhanced External Counter pulsation Therapy and Improvement of Functional Capacity In Chronic Heart Failure Patients: A Randomized Clinical Trial. Acta Med Indones, 2015; 47(4):275-282.

Singh V, Kumari G, Chhajer B, Jhingan AK, Saurabh Dahiya S Evaluation of enhanced external counter pulsation effectiveness on clinical 
profile and health-related quality of life in coronary heart disease patients. IJCRLS, 2018; 7(1):796-805.

Soran O, Inui E, Ochiai A, Naya Y. Two year clinical outcomes after enhanced external counterpulsation (EECP) therapy in patients with refractory angina pectoris and left ventricular dysfunction. Int EECP patient registry, 2006; 97(3):17-20.

Spertus JA, Winder JA, Dewhurst TA, et al. Development and evaluation of the Seattle angina questionnaire: a new functional status measure for coronary artery disease. J Am Coll Cardiol, 1995; 25(2):333341.

Taghadosi M, Arani ZA, Reza Gilasi H, et al. Quality of life in patients with ischemic heart disease. JNMS, 2014; 1(1):19-26.

Thompson DR, Yu C-M, et al. Quality of life in patients with coronary heart disease-I: Assessment Tools. Health Qual Life Outcomes, 2003; 1(42):1-5.

Unnikrishnan R, Mohan Anjana R, Mohan V. Diabetes mellitus and its complications in India. Nat Rev Endocrinol, 2016; 12(6):357-370.

Urano $\mathrm{H}$, Ikeda $\mathrm{H}$, Ueno $\mathrm{T}$, Matsumoto $\mathrm{T}$, et al. Enhanced External Counter pulsation Improves Exercise Tolerance, Reduces ExerciseInduced Myocardial Ischemia and Improves Left Ventricular Diastolic
Filling in Patients With Coronary Artery Disease. J Am Coll Cardiol, 2001; 37(1):93-99.

Wu E, Martensson J, Brostrom A, et al. Enhanced external counter pulsation in patients with refractory angina pectoris: a pilot study with six months follow-up regarding physical capacity and health related quality of life. Eur J of Cardio Nur, 2012; 12(5):437-445.

Xavier D, Pais P, Devereaux PJ, Xie C, et al. Treatment and outcomes of acute coronary syndromes in India (CREATE): A prospective analysis of registry data. Lancet, 2008; 371(9622):1435-1442.

Ziaeirad M, Ziaei GR, Sadeghi N, et al. The Effects of Enhanced External Counter pulsation on Health related Quality Of Life in Patients with Angina Pectoris. Iran J Nurs Midwifery Res., 2012; 17(1):41-46.

\section{How to cite this article:}

Singh V, Kumari G, Chhajer B, Jhingan AK, Dahiya S. A Comparative Assessment to Evaluate Enhanced External Counter Pulsation Effect on Physical Profile and Quality of Life in Diabetic and Non-Diabetic Coronary Heart Disease Patients. J App Pharm Sci, 2018; 8(06): 113-123. 